\title{
Prophetie der Wunde(n) - die Jahreslosung 2017 angesichts von Krieg und Gewalt
}

Ruth Poser

\section{Neues Herz, neuer Geist?}

Mit Ez 36,26: „Gott spricht: Ich schenke euch ein neues Herz und lege einen neuen Geist in euch" hat die Ökumenische Arbeitsgemeinschaft für Bibellesen (ÖAB) für das Jahr 2017 erstmals eine Jahreslosung aus dem ersttestamentlichen Ezechielbuch ausgewählt ${ }^{1}$ - und damit aus einem Buch, das in Theologie und Kirche eher eine Randstellung einnimmt und vielen als besonders schwierig und sperrig gilt. Das hat vor allem mit seiner ungeheuren Gewaltfülle zu tun - und mit dem Umstand, dass Gott selbst in ihm so gewaltverstrickt erscheint. In der feministischen Forschung gelten vor allem die Kapitel 16 und 23, in denen die Stadt Jerusalem als hurerische, von Gott gewalttätig bestrafte Frau ins Bild gesetzt ist, als »texts of terror $\aleph^{2}$, die in der Gefahr stünden, (männliche) Gewalt (gegen Frauen) zu legitimieren. ${ }^{3}$ Einige Forscher_innen attestieren auch den »heilvolleren" Abschnitten gegen Ende des Buches, nicht zuletzt Kap. 36, dem die Jahreslosung entstammt, eine ausgesprochen bedrückende Grundstimmung. Zwar verspreche Ezechiel, »dass Israels Deportierte gesammelt und in das Land Kanaan zurückgebracht werden, um dort für immer in Wohlstand zu leben - aber nicht ein Mal redet er davon als einem Akt der Gnade, des Mitleids, der Vergebung, der Rettung, der Wiedergutmachung, der Freundlichkeit, der Freude, des Bundesvertrauens, der Versöhnung, des Trostes « ${ }^{4}$. Lässt sich die Jahreslosung 2017 also nur als Trost-, Ermutigungs- und Aufbruchs-

\footnotetext{
1 Vgl. hierzu die Informationen auf der Homepage der ÖAB (www.oeab.de [23.11.2016]).

2 Dieser Begriff geht auf die feministische Bibelwissenschaftlerin Phyllis Trible zurück (vgl. dies., Texts of Terror: Literary-Feminist Readings of Biblical Narratives, Philadelphia 1984).

3 Vgl. Ruth Poser, Das Ezechielbuch als Trauma-Literatur, Leiden/Boston 2012, 371-409 (Ez 16), 435-467 (Ez 23).

4 Baruch J. Schwartz, Ezekiel's Dim View of Israel's Restoration, in: Margaret S. Odell/John T. Strong (Hg.), The Book of Ezekiel: Theological and Anthropological Perspectives, Atlanta 2000, 43-67, hier 55 (Übers. Verf.).
} 
wort lesen (ich unterstelle, dass wir sie gerne so lesen wollen), wenn sie aus ihrem Kontext herausgerissen, wenn der Rest des Buches ausgeblendet wird?

M.E. kann derVers Ez 36,26 in seiner Tiefgründigkeit im Gegenteil nur erfasst werden, wenn er im Zusammenhang des Buches und dessen vermutlichen geschichtlichen Hintergrund betrachtet wird: der Belagerung, Eroberung und Zerstörung Jerusalems unter dem babylonischen Herrscher Nebukadnezar 587/86 v.u.Z. und den damit verbundenen Deportationen von Kriegsgefangenen. Wird dieser Hintergrund ernst genommen und konkret gemacht, wird das Ezechielbuch noch einmal anders lesbar: Als literarische Auseinandersetzung mit Kriegsgewalt, die Menschen tatsächlich erlitten haben, als theologische Überlebens- oder Trauma-Literatur. Vor diesem Hintergrund erweist sich die Jahreslosung als "verwundetes" und gerade deshalb konstruktives und wirkmächtiges Wort, das die Ambivalenz von Vulnerabilität als "Risiko und Ressource $\|^{5}$ in besonderer Weise anschaulich macht.

Im Folgenden werfe ich zunächst einen Blick auf den TraumaBegriff, der mir den neueren Vulnerabilitätskonzeptionen nahe zu stehen scheint (2.); daran anschließend erläutere ich meine Perspektive auf das Ezechielbuch als Trauma-Literatur (3.). Abschnitt 4. beschäftigt sich mit dem verwundeten Protagonisten des Buches, dem Ich-Erzähler und Propheten Ezechiel, und mit der Frage, ob und inwiefern dessen »traumatischer Prophetie« überhaupt etwas »theologisch Konstruktives « abzuringen sei. Darauf folgt eine Analyse (der Elemente) der Jahreslosung im Kontext der Ezechielerzählung (5.), bevor in Abschnitt 6. Aspekte der "theanthropologischen " ${ }^{6}$ Relevanz von Ez 36,26 beleuchtet werden. Am Schluss steht ein Ausblick, der die biblische Rede von "Herz» und "Geistkraft» mit weiteren Kontexten "ver-spricht» (7.)

\footnotetext{
5 Heike Springhart, Der verwundbare Mensch. Sterben, Tod und Endlichkeit im Horizont einer realistischen Anthropologie, Tübingen 2016, 211.Vgl. hierzu auch unten Abschnitt 6.

${ }^{6}$ Der Begriff der "Theanthropologie" geht auf Karl Barth (vgl. ders., Einfuihrung in die evangelische Theologie, Zürich 1962,18) zurück. Barth beschreibt mit diesem Begriff die "Aufhebung von Theologie und Anthropologie in eine Geschichte, in der es [...] um die Geschichte Gottes mit den Menschen [geht], aus der allererst der Mensch seine Menschlichkeit gewinnt, dadurch, dass Gott sich ihm als Gott erweist" (Gunda Schneider-Flume, Grundkurs Dogmatik. Nachdenken über Gottes Geschichte, Göttingen 2004, 44).
} 


\section{Trauma bedeutet »Wunde»}

Der Begriff "Trauma« kommt aus dem Griechischen, wo er für "Verletzung, Verwundung, Wunde" steht. In der zeitgenössischen Medizin wird er sowohl für körperliche ("Traumatologie») als auch für seelischeVerletzungen (»Psychotraumatologie») gebraucht. Doch haben verschiedene Trauma-Konzepte inzwischen in nahezu allen Bereichen der Kulturwissenschaften Einzug gehalten. So beleuchten etwa Soziologie und Sozialpsychologie die Folgen von Natur- und Kriegskatastrophen als »kollektive Traumatisierungen«, während im Zusammenhang mit den Berichten und Erzählungen von ShoahÜberlebenden der Zweig der »literarischen Trauma-Theorie» entstanden ist.

Psychische Traumata haben mit völlig überwältigenden äußeren Ereignissen (Natur- oder technische Katastrophen, aber auch menschengemachte Gewalt wie Vergewaltigung, Krieg und Folter) zu tun. Die Betroffenen erleben sich an Leib und Leben als massiv bedroht, als total hilflos und schutzlos preisgegeben. Wenn die auf der Ebene des Stammhirns liegenden Verarbeitungsmöglichkeiten, die bei Gefahr automatisch aktiviert werden, d.h. Flucht- oder Kampfimpulse, nicht greifen, schaltet der Organismus auf den »Erstarrungsmodus (den sogenannten Totstellreflex) um. Dabei sinken Blutdruck und Herzfrequenz, es werden körpereigene Opioide und schmerzausschaltende Hormone ausgeschüttet. Sogenannte höhere Hirnfunktionen, darunter die Funktionen des Gedächtnisses, werden stark eingeschränkt, so dass die lebensbedrohliche Situation nicht rational erfasst und im Nachhinein kaum versprachlicht und zusammenhängend eingeordnet werden kann. (Post-)Traumatische Symptome oder Syndrome entstehen dann, wenn die Gewaltereignisse für die Betroffenen so massiv und einschneidend sind, ${ }^{7}$ dass die

\footnotetext{
7 Nicht jede Person, die mit einem potentiell traumatisierenden Ereignis konfrontiert ist, entwickelt in der Folge eine Posttraumatische Belastungsstörung. Hier spielt neben der Schwere der Ereignisse die (individuell-psychische) Widerstandskraft (Resilienz) die entscheidende Rolle. Dennoch sind Traumatisierungen nicht als »individuelle Probleme« engzuführen; sie stellen vielmehr eine gemeinschaftliche bzw. gesellschaftliche Herausforderung dar, spiegelt sich doch in den (post-)traumatischen Symptomen bzw. Syndromen Einzelner eine umfassende "Pathologie der Wirklichkeit» wider.Vgl. hierzu auch Carolin Emcke, Weil es sagbar ist. Über Zeugenschaft und Gerechtigkeit, Frankfurt a.M. ${ }^{2} 2016$, 23: "Mit dem wachsenden Interesse an der Traumaforschung hat sich der Fokus von der verstörenden Gewalt hin zu dem traumatisierten Opfer der Gewalt verschoben. Als gestört gelten nun oftmals nicht mehr die Strukturen und Praktiken der Entrechtung und Gewalt, sondern die Menschen, die ihr unterworfen wurden."
} 
beschriebenen reflexartigen Reaktionen gleichsam auf Dauer gestellt bleiben. Das Ereignis bzw. die Reaktionen darauf können nicht aus dem Organismus »entlassen werden", sondern besetzen ihn im Sinne einer unsichtbaren schwärenden Wunde, die schwerwiegende Folgen hat:Auf der einen Seite lässt die erlittene Gewalt den Opfern keine Ruhe mehr, drängt sich ihnen etwa in Form von Alpträumen, Flashbacks und Panikattacken immer wieder auf und lässt sie die Situation mit allen Sinnesqualitäten und Affekten wieder und wieder durchleben (»Intrusion"). Auf der anderen Seite versuchen die Betroffenen mit aller verbliebenen Kraft, Angst, Schmerz und Ohnmacht abzuwehren und sich vor allem, was mit dem Gewaltereignis in Zusammenhang steht, zu schützen. Emotionale Erstarrung, psychische Lähmung und Rückzug aus sozialen Bezügen sind die Folge (»Konstriktion«). Hinzu kommen Symptome der Übererregung wie Schlaf- und Konzentrationsstörungen, mangelnde Impulskontrolle und emotionale Labilität. Das Vertrauen in die eigene Person, in andere Menschen, die Welt - in das, was vor dem traumatisierenden Ereignis Halt gegeben hat - trägt nicht mehr. Viele traumatisierte Menschen ringen mit dem Themen Scham und Schuld. Angesichts der Ermordung von Verwandten und Freund_innen fühlen sich manche sogar wegen des eigenen Überlebens schuldig. Manche nehmen - obwohl sie robjektiv < keine Schuld trifft - alle Schuld auf sich, bezichtigen sich selbst an Stelle des Täters. Solche traumatische Schuldübernahme hängt oft damit zusammen, »dass es für das psychische Gleichgewicht leichter erträglich sein kann, schuld gewesen statt völlig ohnmächtig gewesen zu sein«. ${ }^{8}$

\section{Das Ezechielbuch - kriegswund}

Das Ezechielbuch erzählt von Ereignissen aus den Jahren 594 bis 572 v.u.Z. (vgl. die Datumsangaben in 1,2 und 40,1). Seine Hauptfigur, der Priestersohn Ezechiel, ist bereits 597 v.u.Z., als Jerusalem zum ersten Mal von den Babyloniern belagert wurde, zusammen mit weiteren Kriegsgefangenen nach Babylonien verschleppt worden. Inmitten der Deportierten wird er von Gott zum Propheten für Israel bestimmt und soll ihm mitteilen, was Gott vorhat: Gott will das Ende über die "sündige " Hauptstadt Jerusalem kommen lassen. Er malt dieses Ende wieder und wieder in immer noch gewalttätigeren Sprachbildern aus (Ez 1-23). In dem Moment, als sich das angekün-

\footnotetext{
8 Ebd., 32. Zur "Symptomsprache« von Traumata vgl. ausführlich Poser, Ezechiel-
} buch, $57-119$. 
digte Schreckensszenario mit der erneuten Belagerung Jerusalems realisiert (Ez 24,1f.), weitet Ezechiel seine Unheilsbotschaft auf fremde Nationen aus (Ez 25,1-33,20). Der Ton der Prophetie wird erst heilvoller, als ihn die Nachricht von der endgültigen Zerstörung Jerusalems erreicht $(33,21 \mathrm{f}$.). Nun kommen die im Exil Lebenden wieder in den Blick: Den Deportierten wird die Rückkehr ins Land Israel verheißen, wo sie in einem ewigen Bund mit Gott in Frieden leben werden (Ez 33,23-48,35). Doch auch in diesem hinteren Buchteil brechen die grausigen Bilder von Krieg und Zerstörung noch mehrfach auf (Ez 33,23-29; 35; 38-39).

Ich lese das Ezechielbuch, wie es uns heute vorliegt, als eine besondere Art von Prophetie. M.E. geht es in ihm nicht darum, das Volk und die politische Führung angesichts einer heraufziehenden Katastrophe zur Umkehr zu rufen, und eine solche Umkehr unter Bezugnahme auf Gottes Weisung zu eröffnen. Vielmehr bezieht sich die Ezechielprophetie auf die bereits geschehene Katastrophe der Zerstörung Jerusalems unter Nebukadnezar 587/86 v.u.Z. ${ }^{9}$ Sie nimmt die konkret-körperlichen Erfahrungen des antiken Belagerungskrieges und der antiken Massendeportationspraxis in sich auf, von deren ungeheuren Schrecken andere textliche, bildliche und archäologische Quellen ausdrücklich zeugen: Die vom Belagerungskrieg Betroffenen erlebten "Hunger, Seuche und Schwert» (vgl. Ez 7,15), Kriegsgräuel, Folter, Formen sexueller/sexualisierter Gewalt, Plünderung und Brandschatzung. Nicht nur die unmittelbar an den Kampfhandlungen Beteiligten, sondern alle in der Stadt Lebenden kamen dabei mit traumatisierender Gewalt in Berührung (vgl. Ez 9,6). Wer deportiert wurde, hatte darüber hinaus einen mörderischen Gewaltmarsch über Hunderte von Kilometern zu bewältigen, erfuhr das Dahinsiechen und Sterben von Mitdeportierten, die Zerschlagung von Familien und war schließlich zu einem Leben in völliger Fremde gezwungen, in der Regel ohne Hoffnung auf Rückkehr. ${ }^{10}$

Vor diesem Hintergrund lässt sich das Ezechielbuch als Traumaoder Überlebens-Literatur begreifen. Als Erzählung einer traumatisierten Gemeinschaft von nach Babylonien Deportierten und deren

\footnotetext{
9 Über die Datierung des Ezechielbuchs besteht kein Forschungskonsens. Zu den verschiedenen Optionen bzw. Modellen vgl. Rainer Albertz, Die Exilszeit. 6. Jahrhundert v. Chr., Stuttgart u.a. 2001, 260-265. Ich selbst plädiere für eine Datierung jedenfalls weiter Teile der Ezechiel-Erzählung in die Zeitspanne 570 bis 540 v.u.Z., da deren traumatischer Charakter eine gewisse Nähe zu den Schreckensereignissen um 587 v.u.Z. plausibel erscheinen lässt (vgl. Poser, Ezechielbuch, 668-672).

${ }^{10}$ Vgl. hierzu ausführlich Poser, Ezechielbuch, 158-248. Zum antiken (mesopotamischen) Belagerungskrieg vgl. auch Paul B. Kern, Ancient Siege Warfare, Bloomington 1999, 9-85.
} 
Nachkommen hält es all diese Schreckenserfahrungen fest und macht sie erinnerbar. Gleichzeitig eröffnet das Buch einen Raum für die Auseinandersetzung mit diesen Schreckenserfahrungen. Die Kriegskatastrophe soll besprechbar werden und wird besprechbar angesichts Gottes und im Ringen mit Gott.

\section{Ezechiel - ein verwundeter Prophet}

Das Ezechielbuch beginnt mit einer Berufungserzählung, die vor allem durch das Motiv des Überwältigtwerdens Ezechiels durch Gott geprägt ist (Ez 1,1-3,15). Gott beruft Ezechiel nicht in erster Linie - wie dies in anderen prophetischen Erzählungen der Fall ist - als Sprachrohr für seine Botschaft. Vielmehr lässt Gott Ezechiels ganzen Körper zur Botschaft werden. Im Zentrum der Berufungspassage wird Ezechiel von Gott gezwungen, eine über und über mit Klagelauten und Schreckensschreien beschriebene Schriftrolle zu verzehren: ${ }^{11}$

"Reiße deinen Mund auf und iss, was ich dir gebe! Ich schaute - da! - eine Hand war zu mir ausgesandt. Da! - in ihr war eine Schriftrolle. (Gott) breitete sie vor mir aus. Sie war auf Vorderund Rückseite beschrieben. Geschrieben war darauf: Tiefstes Wehklagen, Ach und Weh. Und er sprach zu mir: 'Mensch, was du findest, iss! Iss diese Rolle und geh, rede zum Haus Israel! Ich öffnete meinen Mund, und er ließ mich diese Rolle essen und sprach zu mir: >Mensch, deinen Bauch sollst du essen lassen, deine Eingeweide sollst du füllen mit dieser Rolle, die ich dir gebelk $\mathrm{Da} a ß$ ich sie, und sie wurde in meinem Mund süß wie Honig." (Ez 2,8b-3,3)

Stellt man sich dies leibhaftig vor, wird die Gewaltförmigkeit der Szene deutlich. Drei Aufforderungen Gottes braucht es, bis Ezechiel die riesige, starre, unverdauliche Rolle aus Papyrus, die Gott ihm 3,2 zufolge in den Mund steckt, herunterzuschlucken vermag. Die Aussage "sie wurde in meinem Mund süß wie Honig" beschreibt dabei vielleicht nur den absonderlichen Umstand, dass die Rolle hinuntergewürgt werden kann. Der mittelalterliche Gelehrte Rabbi David Kimchi erläutert den letzten Satz der Szene wie folgt: "So gab ich meinem Bauch zu essen, denn ich spie sie [die Rolle, R.P.] nicht aus. $\|^{12}$

${ }_{11}$ Zum Folgenden vgl. Poser, Ezechielbuch, 345-360.

12 Zitiert nach Moshe Greenberg, Ezechiel 1-20, Freiburg i.Br. u.a. 2001, 96. 
Die körperlichen Folgen der eingezwungenen Mahlzeit werden wenig später deutlich: Ezechiel verliert seine Sprache! Das ist mehr als paradox - denn Gott beauftragt ihn gleich mehrfach, zu seinen Landsleuten zu gehen und mit ihnen zu reden: In Ez 3,1 heißt es: »... und geh, rede zum Haus Israel!«, in 3,4 ganz ähnlich: »... geh, komm zum Haus Israel und rede ...! «, in 3,11 noch einmal ähnlich: »Und geh, komm zu den Verschleppten ... und rede ...! " Das Ende des Beauftragungsgeschehens jedoch wird wie folgt geschildert:

"Wind (ruach) hob mich empor, ergriff mich, und ich ging, bitter, mein Atem (ruach) vergiftet. Die Hand JHWHs war schwer auf mir. Ich kam zu den Verschleppten in Tel Abib, die am Fluss Kebar saßen, und wo sie saßen, da saß ich sieben Tage lang unter ihnen, schreckensstarr.» (Ez 3,14f.)

Während Ezechiel in "Gehen" und »Kommen« der Weisung Gottes entspricht, hat das geforderte Reden keine Entsprechung. An seine Stelle tritt ein schreckensstarres Sitzen; dem neuen Propheten ist, drastisch gesagt, mit derVergiftung des Atems auch die Sprache von Gott "abgewürgt" worden! ${ }^{13}$ Erst an viel späterer Stelle, in einem Zusammenhang, in dem er durch einen Flüchtling von der Zerstörung Jerusalems hört, gewinnt er sie zurück (Ez 33,21f.; vgl. 3,25-27; 24,25-27).

Der Text stellt den Lesenden Ezechiel - ähnlich wie Davids Tochter Tamar (vgl. 2 Sam 13,20) und Hiob (vgl. Ijob 2,12f.) - als gewalttätig Verletzten, als Traumatisierten vor Augen. Als solcher wird er zum Propheten und zum Zeichen für das Haus Israel sowie für die Leser_innen des Textes. Sein Trauma umfasst dabei auch den Sprachverlust, das Hin- und Hergerissensein zwischen Schweigen und Aussprechen, von dem zahlreiche Überlebende extremer Gewalt betroffen sind.

Aber noch etwas anderes ist für die Darstellung von Ezechiels Erleben kennzeichnend: Sein Bewegtwerden durch ruach (zum Begriff vgl. unten). Ez 3,14f. (und weitere Stellen) erzählen davon, dass ruach Ezechiel aufhebt und mitnimmt. Ruach erscheint hier als eine Art "Transportmittel«, das Ezechiel riesige Distanzen überwinden lässt. Während des Berufungsgeschehens wird er, nachdem ihn die Mas-

${ }^{13}$ Vgl. Ijob 6,4 und dazu Jürgen Ebach, Streiten mit Gott. Hiob, Teil 1: Hiob 1-20, Neukirchen-Vluyn 1995, 71. Bei Gregory Y. Glazov, The Bridling of the Tongue and the Opening of the Mouth in Biblical Prophecy, Sheffield 2001, 222, heißt es zur Schriftrollenszene des Ezechielbuchs: »It [the scroll, R.P.] tastes sweet but embitters and inflames, or rather poisons, Ezekiel's spirit, throwing him into a seven-day-long state of silent astonishment or stupefaction [...]." 
sivität der Erscheinung Gottes in die Knie gezwungen hat, zweimal von ruach auf seine Füße gestellt. In 2,2 heißt es (vgl. 3,24): »Als (Gott) zu mir redete, kam ruach in mich - sie richtete mich auf meine Füße. Da hörte ich, was (Gott) zu mir sagte. «Ruach also vermag Ezechiels körperliches Erleben zu verändern. Er erfährt ein Aufgerichtetwerden, das ihn hören, ihn wahr-nehmen lässt. Zunächst ist es noch so, dass der Prophet darin als passiv erscheint - ruach macht mit ihm, was sie will. Doch verändert sich das im Laufe des Buches, wie noch zu sehen sein wird.

Im Anschluss an die Berufungspassage fordert Gott Ezechiel auf, mit und an seinem Körper verschiedene zeichenhafte Handlungen zu vollziehen (Ez 4,1-5,4), die allesamt die Situation der Belagerung, Eroberung und Zerstörung Jerusalems und das Schicksal der dort lebenden Menschen ins Bild setzen (vgl. Ez 5,5-17). Als von Gott dazu Beauftragter ist er hierbei Belagerer und Belagerter zugleich. Mit Stricken gebunden soll er 390 Tage auf der linken und 40 Tage auf der rechten Körperseite liegen und dabei gegen Jerusalem prophetisch reden (Ez 4,4-8). Er soll sich - wiederum kommt das Motiv des Essens (und Trinkens) ins Spiel - von geringsten Rationen an Brot und Wasser ernähren (Ez 4,9-11). So wenig wird ihm zugestanden, dass es zum Überleben nicht reichen und nur grausames Dahinsiechen bedeuten würde. Schließlich trägt Gott ihm auf, seine Kopf- und Barthaare, ein Zeichen der Lebenskraft und männlicher wie weiblicher »Potenz ${ }^{14}$, mit einem scharfen Schwert zu scheren und mit bzw. an den Haaren weitere Kriegshandlungen "durchzuspielen" (Ez 5,1-4). Die Bibelwissenschaftlerin Nancy Bowen, die das Ezechielbuch ebenfalls unter dem Stichwort "Trauma" liest, schreibt hierzu:

"Ezechiels Re-Inszenierungen des traumatischen Falls von Jerusalem [...] ähneln den Handlungen von Opfern, die ein erlittenes Trauma in verschiedenen Formen selbstverletzenden Verhaltens fortgesetzt ausleben. Sein Hungern und sein Essen ekelerregender Nahrungsmittel haben eine erstaunliche Nähe zu verschiedenen Essstörungen, insbesondere zur Anorexie [...]. Der Akt des Sich-Scherens mit einem scharfen Schwert zeigt ein lebensgefährliche Risiken eingehendes Verhalten an. Auch das Auf-der-Seite-Liegen spiegelt ein den eigenen Körper schädigendes Verhalten wider. Solche selbstverletzenden Akte

\footnotetext{
${ }^{14}$ Vgl. hierzu Silvia Schroer/Thomas Staubli, Die Körpersymbolik der Bibel, Darmstadt 1998, 107-114.
} 
können als symbolische oder buchstäbliche Wiederholungen der ursprünglich erlittenen Gewalt begriffen werden. «" ${ }^{15}$

In dem Umstand, dass mehrere der "angedachten« Handlungen mit Hunger und Durst und mit dem Bruch von Essenstabus zu tun haben, spiegeln sich die Lebensmittelknappheit in einer für längere Zeit belagerten Stadt und deren furchtbare Folgen für die Bevölkerung wider, wie sie vermutlich auch für die anderthalbjährige Belagerung Jerusalems durch die Babylonier 589/88-587/86 v.u.Z. prägend waren (vgl. 2 Kön 25,3; Jer 52,6). ${ }^{16}$ In diesen Zusammenhang scheint auch die Ankündigung Gottes in Ez 5,9f. zu gehören, derzufolge der Hunger in Jerusalem schließlich so ungeheuer groß werden wird, dass Eltern ihre Kinder und Kinder ihre Eltern verschlingen. Schlimmeres, so sagt sie selbst, hat die Gottheit Israels ihrem Volk nie angetan. ${ }^{17}$

Mit ähnlich schrecklichen Aufforderungen und Ankündigungen, die allesamt die Schwere der Kriegskatastrophe widerspiegeln, geht es im Ezechielbuch noch lange weiter. Unter Rückbezug auf die Schriftrollenszene schreibt Jürgen Ebach hierzu: »Bevor freilich sSeufzen, Klage und Weh in den Worten Ezechiels süß werden, braucht es lange Zeit. [...] Das Heil muss sich lange durcharbeiten durch das Unheil. ${ }^{18}$ Einige Bibelwissenschaftler_innen allerdings unter ihnen auch Forscher_innen, die Trauma- und postkoloniale Konzeptionen einbeziehen - bestreiten auch, dass im Ezechielbuch überhaupt etwas Heilsames enthalten sei. So kommt etwa Carolin J. Sharp in einem Artikel aus dem Jahr 2011 zu einem wenig hoffnungsvollen Fazit:

»Ezechiel spaltet den `Bundeskörper - Gott und Volk - in eine distanzierte, transzendente Gottheit und ein zutiefst beschämtes und sexualisiertes Juda, das nur überlebt, um die eigene Widerlichkeit zu bezeugen. [...] $>$ Geschichtsschreibung von unten ${ }^{19}$

\footnotetext{
${ }^{15}$ Nancy R. Bowen, Ezekiel, Nashville 2010, 28 (Übers.Verf.).

${ }^{16}$ In 2 Kön 25 und Jer 52 wird die Hungersnot der Bevölkerung als einzige (indirekte) Begründung dafür angeführt, dass es den Babyloniern schließlich gelang, Jerusalem nach eineinhalbjähriger Belagerung "aufzubrechen".

${ }^{17}$ Die Hebräische Bibel erwähnt Anthropophagie (»Kannibalismus») einzig im Kontext von Belagerungskriegen (vgl. Lev 26,29; Dtn 28,52-57; 2 Kön 6,27-29; Jes 9,19; Jer 19,9; Sach 11,9; Klgl 2,20; 4,10) - ein realistischer Hinweis darauf, wie schwerwiegend Hungersnöte in eingeschlossenen Städten werden konnten.

18 Jürgen Ebach, Ezechiel isst ein Buch - Ezechiel ist ein Buch, in: ders., "Iss dieses Buch!«, Theologische Reden 8, Wittingen 2008, 11-24, hier 23.

19 "Geschichtsschreibung von unten" steht der Sozialgeschichte nahe (zu diesem Begriff und zur Sozialgeschichte Israels vgl. Rainer Kessler, Sozialgeschichte des alten
} 
[sollte] nicht als heldenhafte Um-Schreibung der Machtverhältnisse zugunsten der Unterdrückten romantisiert werden [...]. Subalterne ${ }^{20}$ Rede kann Dynamiken der Ausgrenzung reproduzieren und furchtbare neue Ausschlüsse hervorbringen. 'Geschichtsschreibung von unten kann hässlich sein. $\star^{21}$

Ist das Ezechielbuch also doch ein text of terror, dem nichts Heilsames innewohnt, der im Gegenteil Opferbeschuldigung (blaming the victim) betreibt und nur neue, tiefere Wunden schlägt? M.E. ist die "Hässlichkeit» der Ezechielerzählung zu allererst ein Spiegel für die Schwere der traumatischen Katastrophe und deren Folgen, für Gedanken und Gefühle, mit denen Überlebende extremer Gewalt zu kämpfen haben. Die Massivität der Kriegswunde(n) ist hier "todernst" genommen, auch der Umstand, dass vollständige TraumaHeilung zumeist nicht möglich ist. Die extremen Körperbilder, auch das von Juda/Jerusalem als sexualisiertem, feminisiertem Gemeinschaftskörper (vgl. Ez 16; 23), nehmen die Schreckenserfahrungen des antiken Belagerungskrieges, zu denen sexuelle Gewalt und weitere Gräueltaten gehörten, in sich auf. Sie sind darin auch ein Versuch, Sprache zu finden für etwas eigentlich »Unsägliches«.

Die beschriebene Spaltung lässt sich aber darüber hinaus auch im Sinne einer traumatischen Schuldübernahme (s.o.) verstehen, insofern das "Haus Israel« die Katastrophe als Gottes »angemessene» Strafe für eigene politische, soziale und kultische Vergehen deutet. Diese Selbststigmatisierung "rationalisiert" das erlittene Unheil als verhinderbares - hätte Israel der Weisung JHWHs entsprechend gelebt, hätte man dem göttlichen Zorn entgehen können. Das Trauma verliert dadurch sowohl den Charakter der Zwangsläufigkeit als auch der Zufälligkeit; indem Gott in die Täter_in-Rolle gerückt wird,

Israel. Eine Einführung, Darmstadt ${ }^{2} 2008$ [2006]) und meint eine Annäherung an Geschichte, »die keine großen Personen und Handlungsmächte in den Blick nimmt, sondern jene Menschen [...], die ihre Geschichte leben und dabei kaum geschichtsmächtig hervortreten« (Susanne Gorges-Braunwarth, Frauenbilder - Weisheitsbilder Gottesbilder in Spr 1-9: Die personifizierte Weisheit im Gottesbild der nachexilischen Zeit, Münster 2002, 119f.).

${ }^{20}$ Der heute umrungene Begriff "subaltern" geht auf den marxistischen Denker Antonio Gramsci (1891-1937) zurück. In einem weiten Sinn kann er Personen oder Gruppen von untergeordnetem Rang oder "Stand" bezeichnen, wobei die Abwertung oder Ausgrenzung aufgrund von Ethnizität, Klasse, Geschlecht, sexueller Orientierung oder Religiosität vorgenommen wird.

${ }^{21}$ Carolin J. Sharp, Sites of Conflict:Textual Engagements of Dislocation and Diaspora in the Hebrew Bible, in: Brad E. Kelle u.a. (Hg.), Interpreting Exile: Displacement and Deportation in Biblical and Modern Contexts, Atlanta 2011, 365-376, hier 373 (Übers.Verf.). 
verlieren die "weltlichen Täter_innen" (die Babylonier_innen) ihre Macht. In seinem Buch Holy Resilience aus dem Jahr 2014 beschreibt der Bibelwissenschaftler David M. Carr das diesem Erklärungsversuch innewohnende "Überlebenspotential" wie folgt:

"Diese Selbst-Stigmatisierung eröffnete Israel einen Weg, sich in einer Situation der Hilflosigkeit als selbst-wirksam zu erkennen. [...] Das, was viele Leidende noch mehr ängstigt als die Idee, dass Gott sie bestraft, ist die Idee, dass Gott vollkommen abwesend ist [...]. Für einige kann solche Selbst-Stigmatisierung zerstörerisch sein, sie zersetzt ihr Vertrauen. Aber für andere bietet die Idee eines machtvollen Gottes, sogar eines richtenden Gottes, Sicherheit. Es gibt die Möglichkeit, das eigene Verhalten zu verändern und bewahrt zu werden. Wenn die Welt völlig gott-los ist, kann das ganz anders aussehen - dann ist man den gewaltvollsten [innerweltlichen] Kräften unterworfen, auch den brutalsten, tyrannischsten $[\ldots] . \mathbb{1}^{22}$

Führt man diesen Gedanken weiter, bildet die dem »Haus Israel« zugeschriebene Schuld nicht nur die Grundlage, um aus traumatischer Ohnmacht ins Handeln zurückzufinden, sie eröffnet zugleich die Möglichkeit, dass Israels Gottheit die Katastrophe des Exils überlebt und neu zu sich und den Ihren zu kommen vermag. Doch der Preis erscheint (zu) hoch: ein gewalt-nahes, gewalt-volles, vielleicht sogar sadistisches Gottesbild auf der einen, ein massives blaming the victim auf der anderen Seite. Dennoch - zwischen den Zeilen oder im Subtext des Ezechielbuchs gibt es noch Anderes zu entdecken. Diesen gleichsam paradoxen Spuren will ich mich nun zuwenden.

\section{Verwundet sein, verwundbar werden oder: Vulnerabilität als Subtext}

Eine dieser Spuren wird durch die zentralen Elemente der Jahreslosung, »Herz« (hebr. לבב/לב [lew/lewaw]) und "Geist" (hebr. רוח [ruach]) gelegt. Beide Begriffe spielen im Ezechielbuch eine wesentliche Rolle.

Ruach meint von der Grundbedeutung her wahrscheinlich »bewegte Luft" oder "Luft in Bewegung", was sich sowohl auf den "Wind" als auch auf den "Atem» beziehen kann. Insgesamt lassen

${ }^{22}$ David M. Carr, Holy Resilience:The Bible's Traumatic Origins, New Haven/London 2014, 32f. (Übers. Verf.). 
sich drei Dimensionen von ruach unterscheiden: 1. Wind, Bewegungsenergie - spürbare, gewaltige Dynamik, die niederreißen und aufbauen kann; 2. Antriebskraft, Lebensgeist, Atem - das, was Menschen zu lebendigen Wesen macht; und 3. Inspiration, Charisma, Begabung, göttliche "Eingebung« - spirituelle, schöpferische Kraft, die Menschen mit Gottes Wort und göttlicher Lebendigkeit in Verbindung setzt. ${ }^{23}$

"[Ruach] ist eng verwandt mit [räwach] /Weite / Raum<, es wird gebraucht, wenn jemand weit wird, erleichtert ist, aufatmet [...]. [Ruach] schafft Raum, sie setzt in Bewegung, führt aus der Enge in die Weite und macht so lebendig. DieserVorgang ist vor allem beim erleichterten Aufatmen zu beobachten.Von hier aus ist der Bezug zu Lebensatem / Lebenskraft gegeben. $\mathbb{1}^{24}$

Ruach kommt im Ezechielbuch 52 mal vor, wobei sich die genannten Bedeutungsdimensionen kaum voneinander trennen lassen. Gleichzeitig macht ruach im Laufe der Erzählung eine Entwicklung durch, so dass man von einem sich erweiternden Symbol sprechen kann. In der ersten Hälfte des Buches tritt ruach in immer neuen, konstruktiven, aber mehr noch destruktiven Qualitäten in Erscheinung: Ruach bewegt die Räder des göttlichen Thronwagens und sorgt für dessen umfassende Mobilität (vgl. z.B. 1,20). Ruach richtet Ezechiel auf und nimmt ihn mit auf visionäre Reisen, wobei er riesige Distanzen überwindet (vgl. z.B. 2,2; 8,1). Den Bewohner_innen Jerusalems wird die Zerstreuung in alle ruach angekündigt (vgl. z.B. 5,2). Sturmes-ruach wird Mauern zum Einsturz bringen (vgl. 13,1113), Ost-ruach wird das vormals blühende Königtum Judas versengen (vgl. z.B. 19,12). Angesichts der Schrecken des Krieges wird, so heißt es in Kap. 21, jede menschliche ruach erlöschen - die Lebensgeister werden "ausgeblasen" (V12).

Ähnliches ist für den Begriff »Herz« zu beobachten. In der Hebräischen Bibel ist das "Herz" das am häufigsten genannte innere Organ. Es kann das Personzentrum markieren, nicht selten steht es überhaupt für die Mitte bzw. das Innere einer Sache (so ist im Ezechielbuch häufig vom »Herzen des Meeres" die Rede, vgl. z.B. Ez 27,4). Anders als in unserem (Sprach-)Verständnis allerdings zielt der ersttestamentliche Gebrauch nicht allein auf Gefühlsregungen und Stimmungen (wobei auch das vorkommt, vgl. z.B. Ps 4,8), son-

\footnotetext{
${ }^{23}$ Vgl. Jürgen Ebach, Ezechiels Auferstehungsvision (Ez 37), in: BiKi 55 (2000),120126, hier $121 \mathrm{f}$.

${ }^{24}$ Helen Schüngel-Straumann, Art. Geist (AT), in: Das Wissenschaftliche Bibellexikon im Internet (www.wibilex.de), 2009, 2.1. (26.11.2016).
} 
dern auch auf "intellektuelle» Fähigkeiten wie Vernunft, Gedächtnis, Urteilen, Planen und Wollen - Aspekte also, die in unserem Kontext stärker mit Funktionen des Gehirns in Zusammenhang gebracht werden. Bernd Janowski hat das alttestamentliche Herz zuletzt als das menschliche "Beziehungsorgan« bestimmt, »das als Zentrum der Binnenmotivation und Innensteuerung fungiert", wobei "die Gefühle und Absichten" zwar im Inneren des Menschen »ansetzen, sich aber auf die Außenwelt und deren mannigfache Herausforderungen richten ${ }^{25}$

Für lew/lewaw finden sich im Ezechielbuch 47 Belegstellen, wobei vor allem die "Herzensverhärtung« Israels (vgl. Ez 2,4;3,7) sowie das Ausgerichtetsein von dessen Herz auf andere Gottheiten als JHWH angeprangert werden (vgl. z.B. 6,9; 14,3). Mehrfach wird auch die "Herzensüberheblichkeit" fremder Herrscher verurteilt (vgl. z.B. 28,2; 31,10). Während Prophet_innen, die waus ihrem eigenen Herzen" prophezeien, von JHWH gerichtet werden, wird Ezechiel aufgefordert, sein lew/lewaw auf das ihm von Gott Gesagte und Gezeigte auszurichten, es zu verinnerlichen. Auch im Ezechielbuch erscheint das Herz als Beziehungsorgan, als Organ vor allem des Gottesbezugs. Die Schrecken des Krieges, die jede ruach zerstören (s.o.), treffen auch die Herzen: »Es zerschmilzt jedes lew, alle Hände werden schlaff, es erlöscht jede ruach, alle Knie werden Wasser [...] (Ez 21,12; vgl. 21,20; 22,14). Lebensgefährliche Kriegs- und Gerichtssituationen, so kommt hier zum Ausdruck, können zu Handlungsunfähigkeit und Kontrollverlust führen. Das Denken setzt aus, der Atem stockt, Kampf oder Flucht sind nicht mehr möglich. Es ist m.E. keine "Überinterpretation", hier den oben erwähnten traumatischen Erstarrungsmodus beschrieben zu sehen.

Mit dieser Ankündigung verschwindet der ruach-Begriff für einige Kapitel von der "Bildfläche» (eine Ausnahme bildet Ez 27,26). Das "Herz" kommt zwar noch vor, wird aber nicht mehr wie bisher schwerpunktmäßig im Hinblick auf Israels (verfehlten) Gottesbezug verwendet. ${ }^{26}$

\footnotetext{
${ }^{25}$ Bernd Janowski, Das Herz - ein Beziehungsorgan. Zum Personverständnis des Alten Testaments, in: Jürgen van Oorschot/Andreas Wagner (Hg.), Anthropologie(n) des Alten Testaments, Leipzig 2015, 43-64, hier 64.

${ }^{26}$ Es fällt auf, dass lew/lewaw im Ezechielbuch insgesamt "substanzhafter" begriffen wird, während ruach stärker "dynamisch" aufgefasst ist, im Sinne einer (von Gott ausgehenden) Energie, auch wenn die beiden Begriffe gelegentlich fast synonym verwendet zu werden scheinen (vgl. etwa Ez 11,5 [ruach] mit 14,3). Als Organ kommt der lew/lewaw Menschen aller Nationen zu (vgl. z.B. 36,5), während die Belebung durch ruach den Angehörigen des »Hauses Israel« vorbehalten bleibt.
} 
Erst mit Ez 36,26 finden dann beide Begriffe zu neuer Eindeutigkeit, heißt es doch dort: "Ich [JHWH] will euch ein neues lew geben und euer Inneres mit neuer ruach erfüllen. Das steinerne lew will ich aus eurem Körper herausnehmen und euch ein fleischernes lew geben." Anders als in Ez 11,19 (»Ich will ihnen ein einiges lew und neue ruach geben «), anders auch als in Ez 18,31, wo Israel aufgefordert wird, sich selbst "ein neues lew und neue ruach « zu verschaffen, ist dies in Ez 36,26 in direkter Anrede und damit als unmittelbares Beziehungsgeschehen ins Wort gebracht. Auch wird die Verheißung nur hier noch erweitert, indem die neue ruach ausdrücklich als göttliche ruach gekennzeichnet wird: »Meine ruach«, sagt Gott, »will ich in euer Inneres geben und euch zu Menschen machen, die in meinen Bestimmungen gehen und mein Recht bewahren und verwirklichen." $(36,27)$ Es soll also neu werden, was in der traumatischen Katastrophe zerstört worden ist (vgl. Ez 21,12): An die Stelle des erstarrten lew/lewaw ("aus Stein«) tritt ein verlebendigtes (»aus Fleisch «) und somit das, was Denken, Fühlen, Wollen allererst und wieder neu ermöglicht. Die erloschene ruach wird neu "entflammt", so dass das "Haus Israel" neue Lebensenergien entwickeln und wieder in Bewegung - in eine JHWH gemäße Bewegung entsprechend den göttlichen Weisungen - kommen kann.

Das große Versprechen von Ez 36,26 ist eingebunden in die Vision eines umfassenden Veränderungs- und Erneuerungsprozesses, zu dem schließlich auch die Auseinandersetzung mit der erlittenen Kriegskatastrophe gehört (Ez 36,22-38). Erinnerungsarbeit, die Zukunft eröffnet, soll möglich werden. Dabei weiß die Ezechielerzählung auch darum, dass es für solche Erinnerungsarbeit einen geschützten Raum braucht, dass also die unmittelbare tödliche Bedrohung erst einmal vorbei sein muss. Entsprechend verspricht Gott in Ez 36 das Ende des Hungers (V29f.), den Wiederaufbau der in Trümmern liegenden Städte (V33-36) und das Wieder-zahlreichWerden des Volkes Israel (V37f.) und zeichnet sich dabei selbst in konstruktiven Rollen: als Gärtnerin, als Baumeister, als Hebamme. Diese neuen resilienten Aspekte, die in diesen Gottesbildern und in den Bildern des empowerment ${ }^{27}$ des "Hauses Israel « zum Ausdruck kommen, stehen in Ez 36 neben dem, was (noch) traumatisch bleibt - einer Vorstellung von Gott, der nicht um Israels willen, sondern nur um seiner selbst willen handelt (V22f.32), und einer Vorstellung vom »Haus Israel«, das dauerhaft stigmatisiert, zu Scham- und

\footnotetext{
${ }^{27}$ Unter empowerment wird im medizinisch-therapeutischen Kontext die (Förderung der) Befähigung zu selbständigem/selbstbestimmtem Handeln und damit verbunden die Wiedererlangung des Gefühls der "Selbstwirksamkeit» verstanden.
} 
Schuldgefühlen »verdammt« bleibt (V31f.). Auch das plastische Bild der "Herztransplantation" deutet einmal mehr auf einen langen und schmerzhaften Prozess hin, der neben neuen Lebensmöglichkeiten sicher auch neue Schmerzen und Wunden (und später Narben) nach sich zieht. Darin ist es sehr "wahrhaftig". Wichtig erscheint mir in diesem Zusammenhang, dass es sich um eine "gemeinschaftliche Operation " handelt (das "Haus Israel " wird durchgehend im Plural angesprochen) - es geht also um ein kollektives Geschehen, in dem Leid und Schmerz geteilt werden können, das Solidarität und gegenseitige Unterstützung aus sich heraussetzen könnte.

In Ez 37,1-14, d.h. im unmittelbaren Anschluss an den soeben besprochenen Textabschnitt, kommt es zu einer nochmaligen Verdichtung des ruach-Geschehens. Zehnmal kommt das Wort ruach allein innerhalb dieses Textabschnitts vor. In der berühmten Szene von der Wiederbelebung des Totenfeldes wird die zuvor ergangene Verheißung der Gabe neuer ruach visionär umgesetzt und damit erzählerisch erfüllt.Viele der im Vorfeld mit ruach verbundenen Bilder werden hier noch einmal aufgerufen und in ein über die bisherigen Ereignisse hinausweisendes Geschehen integriert. Das Herz hingegen wird in dieser Passage nicht noch einmal eigens erwähnt.

Was genau passiert? Ezechiel wird von Gott und "in Gottes ruach « auf eine Ebene voller völlig vertrockneter Knochen gebracht. In diesem Ausgangsbild wird die furchtbare Realität des Krieges noch einmal anschaulich: Das Szenario erinnert an ein verlassenes Schlachtfeld, auf dem die Getöteten liegengelassen wurden, nicht einmal mehr begraben werden konnten. Die Bezeichnung "Erschlagene" in V9 ist ein Begriff, der im Vorfeld von Ez 37 für den Kriegstod der Einwohner_innen Jerusalems verwendet wurde (Ez 9,6; 21,16; 23,47). Auch über das Motiv der Knochen werden Sprachbilder aus den Gerichtsszenen des Buches aufgenommen (Ez 6;24). Die Rede von den sehr trockenen Gebeinen erinnert an die versengende, austrocknende Wirkung der Ost-ruach, die zuvor zur Beschreibung des Schicksals Israels ins Bild gesetzt worden war.

Doch dieser potenzierten Todeswirklichkeit will Gott nun neues Leben einhauchen. Er tut dies unter Ezechiels Vermittlung, der prophetisch reden und den trockenen Knochen mitteilen soll, dass Gott dabei ist, ruach in sie kommen zu lassen (V5). Als Ezechiel seinen Redeauftrag ausfuhrt, kommt es zu einem Beben, das erstaunlicherweise konstruktive Wirkung hat: Die Knochen rücken aneinander, erhalten Sehnen und Muskelfleisch und werden mit Haut überzogen - der Verwesungsvorgang eines Menschen- oder Tierkörpers wird in sein genaues Gegenteil verkehrt. Doch den 
derart wiederhergestellten Körpern fehlt noch etwas, das von Gott Angekündigte hat sich noch nicht vollständig erfüllt. »Aber es war keine ruach in ihnen", heißt es in V8. Diese Feststellung ruft noch einmal das "Erlöschen jeder ruach «, von dem in Ez 21,12 die Rede war, ins Gedächtnis.

In Ez 37,9f. kommt es zum auf die »Geistkraft« bezogenen Höhepunkt der Textpassage:

„Er aber sprach zu mir: Künde auf den Geistbraus zu, künde, Menschensohn, sprich zum Geistbraus: So hat mein Herr, ER, gesprochen: Von den vier Brausewinden, Geistbraus, komm, wehe diese Erwürgten an, daß sie leben! Ich kündete, wie er mir geboten hatte. Der Geistbraus kam in sie ein, sie lebten. Sie standen auf ihren Füßen, ein sehr sehr großes Heer." (Ez 37,9f. ${ }^{28}$ )

Aus allen Himmelsrichungen - und die Himmelsrichtungen heißen hier ruchot, werden also, was selten vorkommt, mit dem Plural von ruach bezeichnet - wird die ruach mit ihren energetischen Kräften auf den Punkt zusammengeholt und in den Dienst des empowerment der "Zerschlagenen" gestellt. Ruach erscheint darin als das schöpferische Prinzip, der Lebenshauch, von dem alles Leben abhängt. Der menschliche Atem, der der an sich toten Materie des Leibes Lebendigkeit schenkt, ist nichts anderes als Gottes ruach, Gottes Geistkraft (vgl. Gen 2,7; Ps 104,29f.). Sie richtet die Menschen in umfassendem Sinn auf. Die Auf(er)steh(ungs)geschichte Ez 37 ist eine Schöpfungs- oder Neuschöpfungserzählung.

Gott haucht die ruach nicht selbst ein, sondern beauftragt seinen Propheten Ezechiel mit der Weitergabe der Geistkraft, was sich als Sprachereignis vollzieht. Im Anschluss an Ez 2,2 und 3,24, wo Ezechiel unter Einwirkung der ruach in den Stand versetzt wurde, gibt er nun weiter, was er selbst erfahren hat. Als ein von ruach $\mathrm{Ge}-$ tragener wird er zu deren Vermittler. Der zeichenhafte Prophet, das zeichenhafte "Menschenkind $\aleph^{29}$ Ezechiel ist über die Herbeirufung der Geistkraft an der Entstehung neuen Menschseins beteiligt. Und umgekehrt kann man sagen: Gottes Geistkraft vermittelt sich durch das prophetische Wort, wie es uns aus den biblischen Schriften entgegenkommt.

Die Rede von dem »sehr, sehr großen Heer" setzt der Todesszenerie des Anfangs ("sehr" viele Knochen, "sehr" trocken) eine potenzierte Lebensfülle entgegen. Die Wirksamkeit des propheti-

${ }^{28}$ Übersetzung: Martin Buber/Franz Rosenzweig, Die Schrift, Stuttgart 1992 [1958].

29 "Mensch" oder "Menschenkind» (ben-adam) kommt als JHWHs Anrede an Ezechiel im Ezechielbuch 93 mal vor. 
schen Wortes in der ruach Gottes wird eindrucksvoll unterstrichen. Allerdings wird die Realität des Todes, die im Ezechielbuch immer die Realität des gewaltsamen Kriegstodes ist, dabei nicht verleugnet. Gerade im Bild des Heeres bleibt sie ausgesprochen präsent. Johannes Schnocks schreibt hierzu:

"Die Vision lehnt sich mit ihrer Terminologie im Blick auf Tod und Leben in breiter Entsprechung zu anderen Texten des Ezechielbuches an das sprachliche Inventar von Kriegssituationen an und greift zu Extrembegriffen. $>$ Todk ist das stets gewaltsame Sterben von Kriegsopfern, kurz: die Leblosigkeit von Erschlagenen, während 'Leben` in derVitalität des aktionsbereiten Heeres gipfelt. $\mathbb{1}^{30}$

Gott selbst deutet das Aufstehen der toten Gebeine in V11-14 als Befreiung Israels aus Hoffnungslosigkeit und Todesnähe und als Möglichkeit eines politischen Neuanfangs im verheißenen Land, durch die Wiedervereinigung der verlorenen Stämme des Nordund Südreichs. Einig Israel wird, so die göttliche Verheißung, unter dem messianischen König David im Lande Jakobs leben - unter Verwirklichung der Tora, in einem dauerhaften Friedensbund mit Gott, mit Gottes Heiligtum in der Mitte (Ez 37,15-28).

Mit der eindrucksvollen Verdichtung des ruach-Geschehens im Verlauf von Ez 37 wird die ruach, die im Vorfeld eine schwer einzuordnende und teils auch zerstörerische Größe war, zum Zeichen der neuen Verbindung zwischen der Gottheit Israels und ihrem Volk. Ruach wird zum Ermöglichungsgrund und zum Erkennungszeichen des neuen Bundes. Kaum zufällig findet sich im Zusammenhang der angesprochenen Verheißungen die sog. Bundesformel: "Ihr werdet zum Volk für mich werden, und ich, ich werde für euch Gott sein". Diese neue Verbundenheit macht auch Ez 39,29 noch einmal deutlich, wo es feststellend heißt: »Ich werde mein Angesicht nicht mehr vor ihnen verbergen, denn ich habe meine Geistkraft über das Haus Israel ausgegossen - Ausspruch JHWHs, mächtig über allen."

\footnotetext{
${ }^{30}$ Johannes Schnocks, Rettung und Neuschöpfung. Studien zur alttestamentlichen Grundlegung einer gesamtbiblischen Theologie der Auferstehung, Göttingen 2009, $210 f$.
} 


\section{Verwundete Theologie - Theologie der Verwundbarkeit}

Wie ist die Jahreslosung 2017 theologisch zu deuten, wenn man sie im Zusammenhang des Ezechielbuchs und der Wunde(n), die dieses Buch prägt (prägen), liest? Ich möchte sechs Punkte benennen, die mir besonders wichtig erscheinen:

- Lebendigwerden, Aufstehen, Auferstehen - das ist kein jenseitiges Geschehen. Im Gegenteil: Gott geht - und führt - mitten in die Welt, in die Realität von Gewalt, Krieg und Tod hinein. Das Bild von der Ebene voller vertrockneter Knochen, die an ein verlassenes Schlachtfeld erinnert, macht dies eindrücklich greifbar. Die Belebung der leblosen Körper, die als Teilhabe an der Lebendigkeit Gottes gedacht wird, erfolgt inmitten der Todesstrukturen und zielt auf gemeinschaftliches Leben im Shalom Gottes.

- Gott haucht die ruach nicht selbst ein, sondern beauftragt seinen Propheten, diese Lebens-Gabe zu spenden, was sich als Sprachereignis vollzieht. Ezechiel vermag das Aufgerichtetwerden durch ruach weiterzugeben, das er am eigenen Leibe erfahren hat. Lebendigwerden geschieht durch das die Weisung Gottes aktualisierende prophetische Wort, das - damals wie heute - unter menschlicher Vermittlung zu Gehör gebracht wird. Neben dem Wieder-sprachfähig-Werden geht es um Hören-Können und -Wollen - Hören auch und vor allem auf die von Krieg und Gewalt Gezeichneten.

- Die Gabe der ruach geschieht an jedem und jeder Einzelnen und zielt zugleich auf gelingende weltlich-politische Gemeinschaft. Es sind die konkreten, durch Kriegsgewalt ununterscheidbar zerstörten Körper, die zusammengefügt und belebt werden. Ich verstehe dies als ein Hoffnungszeichen dafür, dass Gott jede einzelne Lebensgeschichte, auch die gewaltsam und unzeitig abgebrochene, aufhebt, in sich birgt und zu Recht bringen wird. Die Lebensgeschichten der Einzelnen bleiben dabei eingebunden in die Geschichte des Volkes Israel inmitten derVölker. Auch diese Geschichte wird die Gottheit Israels, so die Verheißung, zu Recht bringen - indem sie Menschen Anteil gibt an ihrer Lebendigkeit, die Tod und Todesstrukturen überwindet.

- Tragfähigkeit und Überzeugungskraft gewinnt das Symbol der ruach vor allem daraus, dass es Gegensätzliches in sich zu bergen vermag. Es braucht geraume Zeit, bis (die) ruach als Lebenszeichen eindeutiger erkennbar wird. Zunächst erscheint sie widersprüchlich und chaotisch, so dass sie kaum als eine Größe auszumachen ist. Wie hierin von traumatischer Sinn- und Erfahrungszersplitterung erzählt wird, so schildert Ez 37,1-14 die Wiedergewinnung einer Sinnpers- 
pektive. Hier werden alle ruach-»Fragmente « zusammengerufen und in den Dienst des empowerment der lebendig-toten Überlebenden der Katastrophe (V11) gestellt. Über das prophetische Wort haben nicht nur die Erzählfiguren Anteil an diesem empowerment, sondern alle, die dieses prophetische Wort hören und lesen. Die ruach steht damit auch dafür, dass es die Auseinandersetzung mit der Katastrophe braucht und dass Erinnerung, so schmerzhaft sie auch sein mag, Heilung ermöglichen, Zukunft eröffnen kann.

- Für die Auseinandersetzung mit der traumatischen Katastrophe von 587/86 v.u.Z. bot sich das ruach-Motiv mit seiner Grundbedeutung der "Luft in Bewegung" wohl vor allem wegen seiner Vieldimensionalität und seiner für alle am eigenen Leibe nachvollziehbaren Erlebensnähe (Atem, Wind) an. Über den ruach-Begriff (und dies gilt auch für das Herz-Motiv) lassen sich individuelle und kollektive Erfahrungen von Gewalt, Zerstörung, Tod und Erfahrungen des Heilwerdens, der Wiederbelebung, des Neuanfangs ins Wort bringen. Für das Ezechielbuch als theologischer Traumaliteratur nicht minder entscheidend ist vielleicht, dass über das Motiv der Geistkraft göttliche und weltliche Sphäre miteinander verknüpft werden. Wie die Gottheit Israels selbst durch Geistkraft zur bewegten Bewegerin wird, wie sie über ihre ruach Weltgeschehen schöpferisch in Bewegung setzt, so soll auch ihr Volk durch ihre Kraft neu in Bewegung kommen: auf den Weg der Tora, auf den Weg ins Leben, auf den Weg in eine Welt des Shalom.

- Die Restituierung von lew/lewaw und ruach, die in Ez 36,26 ins Bild gesetzt ist, lässt sich - das mag zunächst paradox klingen auch als auf Gottes Initiative zurückgehende Erneuerung von Verwundbarkeit/Vulnerabilität begreifen. Während weite Teile des Ezechielbuchs Erfahrungen der (tödlichen) Bedrohung und Gefährdung menschlichen Lebens aufgrund physischer und psychischer Verletzlichkeit in den Vordergrund rücken, wird mit Ez 36,26 eine Verletzlichkeit stark gemacht, die "Bedingung für Vertrauen, Empathie und persönliche Beziehungen" und damit "wesentlicher Faktor für respektvollen Umgang, Vertrauen und Sensibilität anderen gegenüber « ist. ${ }^{31}$ Die Geschichte von Herz und Geistkraft, die das Ezechielbuch schreibt, erweist Vulnerabilität als gleichsam "unabdingbare Ressource ${ }^{32}$ gelingender Beziehung - zur Welt, zu anderen Menschen und zu Gott.

\footnotetext{
${ }^{31}$ Springhart, Der verwundbare Mensch, 211.

${ }^{32}$ Ebd.
} 


\section{Herzberührung}

Schließen möchte ich mit etwas, das mich sehr verwundert: Das Ezechielbuch scheint implizit darum zu wissen, wie wichtig körperliche Prozesse im Kontext von Trauma-Heilungsprozessen sind - etwas, das neben Themen wie "Sprache finden", "Integration durch Erzählen", "Notwendigkeit von Zeug_innenschaft" und "imaginativer Arbeit« im Kontext der Psychotraumatologie in den letzten Jahren zunehmend entdeckt wird. Körpertherapien wie Somatic Experien-

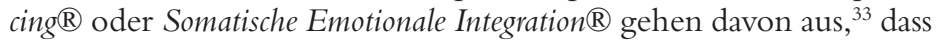
der Organismus die Stress-Reaktionen, die in (post-)traumatischen Symptomen oder Syndromen auf Dauer gestellt sind (s.o.), im Rahmen eines Zeit lassenden, behutsamen Beziehungsgeschehens aus sich heraussetzen kann. Sowohl die (therapeutische) Herzberührung als auch die Unterstützung des Aufatmen(-Könnens) - beides Aspekte, die, jedenfalls indirekt, in der Jahreslosung angesprochen werden - können dabei von großer Wichtigkeit und Wirksamkeit sein. ${ }^{34}$ In ihrem Gedicht "Tröstung" malt Hilde Domin (1909-2006), die selbst immer wieder über die Wunde des Exils und das Wunder des Überlebens geschrieben hat, diesen Prozess schmerzhaft-heilsamer Herzberührung auf beeindruckende Weise aus:

\footnotetext{
${ }^{33}$ Vgl. hierzu die Homepages unter www.somatic-experiencing bzw. www.traumaheilung.de (30.11.2016).

${ }^{34}$ Diesen Hinweis verdanke ich Dipl.-Päd. Ute Lörchner, die beim Frauenfriedenstag am 11.11.2016 einen Vortrag unter der Überschrift "Auswirkungen von Trauma Wege zurück ins Leben« gehalten hat.
} 


\author{
Tröstung \\ Das blasse \\ beschädigte Herz \\ wird aufgenommen \\ und in die frühe Sonne gelegt \\ auf den Brustfedern \\ kleinerVögel. \\ Morgenlotionen \\ aus Blau \\ werden täglich erneut \\ bis die Tränenkrusten \\ weggeschwemmt sind \\ und das Herz \\ schwer wird von Süße \\ wie eine gezuckerte Frucht. \\ Dann wird es eingepflanzt \\ wie Saatkorn aus Gräbern \\ in die schmerzende Furche \\ und die Wunde wird mit dem Speichel \\ sanfter Küsse verheilt. \\ Und das Korn \\ totes Glück \\ schlägt Wurzel und keimt. \\ Alle Adern schmecken danach \\ bis meine Fingerspitzen \\ rosig sind \\ wie die eines Kinds. ${ }^{35}$
}

- Dr. theol. Ruth Poser ist wissenschaftliche Mitarbeiterin für Altes Testament am Fachbereich Evangelische Theologie der Universität Marburg. Ihre derzeitigen Arbeitsschwerpunkte sind »Exegese und Trauma" und "Schamdiskurse in ausgewählten Psalmen«.

${ }^{35}$ Hilde Domin, Sämtliche Gedichte, hg. v. Nikola Herweg/Melanie Reinhold, Frankfurt a.M. ${ }^{4} 2010,220 f$. 